\title{
Question Answering over Curated and Open Web Sources
}

\author{
Rishiraj Saha Roy \\ rishiraj@mpii.de \\ MPI for Informatics, Germany
}

\author{
Avishek Anand \\ anand@13s.de \\ L3S Research Center, Germany
}

\begin{abstract}
The last few years have seen an explosion of research on the topic of automated question answering (QA), spanning the communities of information retrieval, natural language processing, and artificial intelligence. This tutorial would cover the highlights of this really active period of growth for QA to give the audience a grasp over the families of algorithms that are currently being used. We partition research contributions by the underlying source from where answers are retrieved: curated knowledge graphs, unstructured text, or hybrid corpora. We choose this dimension of partitioning as it is the most discriminative when it comes to algorithm design. Other key dimensions are covered within each sub-topic: like the complexity of questions addressed, and degrees of explainability and interactivity introduced in the systems. We would conclude the tutorial with the most promising emerging trends in the expanse of QA, that would help new entrants into this field make the best decisions to take the community forward. Much has changed in the community since the last tutorial on QA in SIGIR 2016, and we believe that this timely overview will indeed benefit a large number of conference participants.
\end{abstract}

\section{MOTIVATION}

\subsection{Background}

Over several decades, the field of question answering (QA) grew steadily from early prototypes like BASEBALL [38], through IBM Watson [37] and all the way to present-day integration in virtually all personal assistants like Siri, Cortana, Alexa, and the Google Assistant. In the last few years though, research on QA has well and truly exploded: this has often resulted in top conferences regularly creating submission tracks and presentation sessions dedicated to this topic. This tutorial will try to highlight key contributions to automated QA systems in the last three to four years coming from the perspectives of information retrieval (IR) and natural language processing (NLP) [16, 19, 20, 28, 39, 56, 63, 65, 76, 84, 88].

\subsection{Perspectives}

In Information Retrieval, QA was traditionally treated as a special use case in search [85], to provide crisp and direct answers to certain classes of queries, as an alternative to ranked lists of documents that users would have to sift through. Such queries, with

Permission to make digital or hard copies of all or part of this work for personal or classroom use is granted without fee provided that copies are not made or distributed for profit or commercial advantage and that copies bear this notice and the full citation on the first page. Copyrights for components of this work owned by others than ACM must be honored. Abstracting with credit is permitted. To copy otherwise, or republish, to post on servers or to redistribute to lists, requires prior specific permission and/or a fee. Request permissions from permissions@acm.org.

SIGIR '20, July 25-30, 2020, Xi'an, China

(C) 2020 Association for Computing Machinery.

ACM ISBN 978-x-xxxx-xxxx-x/YY/MM ...\$15.00

https://doi.org/10.1145/nnnnnnn.nnnnnnn objective answers, are often referred to as factoid questions [21, 23] (a term whose definition has evolved over the years). Factoid QA became very popular with the emergence of large curated knowledge graphs (KGs) like YAGO [74], DBpedia [8], Freebase [13] and Wikidata [86], powerful resources that enable such crisp question answering at scale. Question answering over knowledge graphs or equivalently, knowledge bases (KG-QA or KB-QA) became a field of its own, that is producing an increasing number of research contributions year over year $[12,19,30,63,73,84,88]$. Effort has also been directed at answering questions over Web tables [45, 62], that can be considered canonicalizations of the challenges in QA over structured KGs.

In contrast, QA (in one of the major senses as we know it today) in Natural Language Processing started with the AI goal of whether machines can comprehend simple passages [16, 20, 65, 96] so as to be able to answer questions posed from the contents of these passages. Over time, this machine reading comprehension (MRC) task became coupled with the retrieval pipeline, resulting in the so-called paradigm of open-domain $Q A[16,28,87]$ (a term that is overloaded with other senses as well $[2,33])$. Nevertheless, this introduction of the retrieval pipeline led to a revival of text$Q A$, that had increasingly focused on non-factoid QA [22, 95] after the rise of structured KGs. This has also helped bridge the gap between text and KG-QA, with the latter family gradually incorporating supplementary textual sources to boost recall [71, 75, 76]. Considering such heterogeneous sources may often be the right choice owing to the fact that KGs, while capturing an impressive amount of objective world knowledge, are inherently incomplete.

Terminology. In this tutorial, we refer to knowledge graphs and Web tables as the curated Web, and all unstructured text available online as the open Web.

Content. All material for this tutorial is publicly available at our website https://www.avishekanand.com/talk/sigir20-tute/.

\section{OBJECTIVES}

As mentioned in the beginning, the importance of QA has been fuelled to a large extent by the ubiquity of personal assistants: this has also helped bring together these seemingly independent research directions under one umbrella through a unified interface. One of the goals of this tutorial is to give the audience a feel of these commonalities: this can have a significant effect on overcoming the severely fragmented view of the QA community.

What do we not cover? QA over relational databases is closely related to the independent field NLIDB (natural language interfaces to databases) [53] and is out of scope of this tutorial. Associated directions like Cloze-style QA [52] or specialized application domains like biomedical QA [60] will be mentioned cursorily. Approaches for visual and multimodal QA [40] are out of scope, and so is community question answering (CQA) where the primary 
goal is to match experts with pertinent questions: the answering itself is not by the machine but by humans.

\section{RELEVANCE TO THE IR COMMUNITY}

Related tutorials. A tutorial on QA is not really new to SIGIR: the previous one was presented in 2016 by Wen-tau Yih and Hao Ma [98] (also at NAACL 2016 [97], by the same authors). Textbased QA tutorials appeared way back in NAACL 2001 (Sanda Harab and Dan Moldovan) [41] and EACL 2003 (Jimmy Lin and Boris Katz) [54]. Tutorials on IBM Watson [6,36] and entity recommendation [58] have also touched upon QA in the past. Recent workshops on various aspects of QA have been organized at top-tier conferences: MRQA (EMNLP-IJCNLP 2019), RCQA (AAAI 2019), HQA (WWW 2018), and OKBQA (COLING 2016).

Need for a new one. The unparalleled growth of QA warrants a new tutorial to cover recent advances in the field. Primarily a direction dominated by template-based $[2,83]$ approaches, QA now includes a large number of neural methods [16, 17, 20,44], graphbased $[56,57]$ methods, and even a handful that explore reinforcement learning $[25,61,63]$. Across sub-fields, more complex questions are being handled, complexity being defined in terms of entities and relationships present $[56,84,96]$. Systems are moving from their static counterparts to more interactive ones: an increasing number of systems are including scope for user feedback $[2,50,100]$ and operate in a multi-turn, conversational setting [19, 61, 68, 73]. Interpretability or explainability of presented answers is yet another area of significance $[1,69,79,88]$, as the role of such explanations is being recognized both for developers and end-users towards system improvement and user satisfaction. The tutorial will emphasize each of these key facets of QA. In addition, a summary of the available benchmarks $[3,11,18,19,65,68,80,96]$ in each of the QA sub-fields will be provided, that would be very valuable for new entrants to get started with their problem of choice.

\section{TOPICS}

\subsection{QA over Knowledge Graphs}

The advent of large knowledge graphs like Freebase [13], YAGO [74], DBpedia [8] and Wikidata [86] gave rise to QA over KGs (KG-QA) that typically provides answers as single or lists of entities from the KG. KG-QA has become an important research direction, where the goal is to translate a natural language question into a structured query, typically in the Semantic Web language SPARQL or an equivalent logical form, that directly operates on the entities and predicates of the underlying $\mathrm{KG}[12,19,63,84,88]$. KG-QA involves challenges of entity disambiguation and, most strikingly, the need to bridge the vocabulary gap between the phrases in a question and the terminology of the KG. Early work on KG-QA built on paraphrase-based mappings and query templates that involve a single entity predicate $[11,47,83,93,94]$. This line was further advanced by $[4,9,10,42]$, including the learning of templates from graph patterns in the KG. However, reliance on templates prevents such approaches from robustly coping with arbitrary syntactic formulations. This has motivated deep learning methods with CNNs and LSTMs, and especially key-value memory networks [17, $44,82,91,92]$.
A significant amount in this section of the tutorial will be on answering complex questions with multiple entities and predicates. This is one of the key focus areas in KG-QA now [12, 15, 30, 43, 46, $56,63,84]$, where the overriding principle is often the identification of frequent query substructures. Web tables represent a key aspect of the curated Web and contain a substantial volume of structured information [31]. QA over such tables contains canonicalized representatives of several challenges faced in large-scale KG-QA, and we will touch upon a few key works in this area $[45,62,77]$.

\subsection{QA over Text}

4.2.1 Early efforts. Question answering has originally considered textual document collections as its underlying source. Classical approaches $[67,85]$ extracted answers from passages and short text units that matched most cue words from the question followed by statistical scoring. This passage-retrieval model makes intensive use of IR techniques for statistical scoring of sentences or passages and aggregation of evidence for answer candidates. TREC ran a QA benchmarking series from 1999 to 2007, and more recently revived it as the LiveQA [5] and Complex Answer Retrieval (CAR) tracks [29]. IBM Watson [37] extended this paradigm by combining it with learned models for special question types.

4.2.2 Machine reading comprehension (MRC). This is a $\mathrm{QA}$ variation where a question needs to be answered as a short span of words from a given text paragraph $[65,96]$, and is different from the typical fact-centric answer-finding task in IR. Exemplary approaches in MRC that extended the original single-passage setting to a multi-document one can be found in DrQA [16] and DocumentQA [20] (among many, many others). Traditional fact-centric QA over text, and multi-document MRC are recently emerging as a joint topic referred to as open-domain QA $[28,55,87]$.

4.2.3 Open-domain QA. In NLP, open-domain question answering is now a benchmark task in natural language understanding (NLU) and can potentially drive the progress of methods in this area [51]. The recent reprisal of this task was jump-started by QA benchmarks like SQuAD [65] and HotpotQA [96], that were proposed for MRC. Consequently, a majority of the approaches in NLP focus on MRC-style question answering with varying task complexities [27, $32,51,81]$. This has lead to the common practice of considering the open-domain QA task as a retrieve and re-rank task. In this tutorial, we will introduce the modern foundations of open-domain QA using a similar retrieve-and-rank framework. Note that our focus will not be on architectural engineering but rather on design decisions, task complexity, and the roles and opportunities for IR.

\subsection{QA over Heterogeneous Sources}

Limitations of QA over KGs has recently led to a revival of considering textual sources, in combination with $\mathrm{KGs}$ [71, 72, 75, 76, 92]. Early methods like PARALEX [34] and OQA [35] supported noisy KGs in the form of triple spaces compiled via Open IE [59] on Wikipedia articles or Web corpora. TupleInf [49] extended and generalized the Open-IE-based PARALEX approach to complex questions, and is geared for multiple-choice answer options. TAQA [99] is another generalization of Open-IE-based QA, by constructing a KG of $n$-tuples from Wikipedia full-text and question-specific 
search results. SplitQA [80] addressed complex questions by decomposing them into a sequence of simple questions, and relies on crowdsourced training data. Some methods for hybrid QA start with KGs as a source for candidate answers and use text corpora like Wikipedia or ClueWeb as additional evidence [26, 75, 76, 79, 89, 92], or start with answer sentences from text corpora and combine these with KGs for giving crisp entity answers [71, 78].

\subsection{New Horizons in QA}

4.4.1 Conversational QA. Conversational QA involves a sequence of questions and answers that appear as a natural dialogue between the system and the user. The aim of such sequential, multi-turn QA is to understand the context left implicit by users and effectively answer incomplete and ad hoc follow-up questions. Towards this, various recent benchmarks have been proposed that expect answers that are boolean [69], extractive [18] and free-form responses [68], entities [19], passages [48], and chit-chat [101]. Leaderboards of the QuAC [18] and CoQA [68] datasets point to many recent approaches in the text domain. Recently, the TREC CAsT track [24] (http://www.treccast.ai/) and the Dagstuhl Seminar on Conversational Search [7] tried to address such challenges in conversational search. For KG-QA, notable efforts include [19, 39, 70, 73]. We will focus on the modelling complexity of these tasks and a classification of the approaches involved.

4.4.2 Feedback and interpretability. Static learning systems for QA are gradually paving the way for those that incorporate user feedback. These mostly design the setup as a continuous learning setup, where the explicit user feedback mechanism is built on top of an existing QA system. For example, the NEQA [2], QApedia [50], and IMPROVE-QA [100] systems primarily operate on the core systems of QUINT [4], DrQA [16], and gAnswer [42], respectively. A direction closely coupled with effective feedback is the interpretability of QA models, that is also essential to improving trust and satisfaction $[1,88]$. This section is for experts and we will discuss potential limitations and open challenges.

4.4.3 Clarification questions. A key aspect of mixed-initiative systems [64] is to be able to ask clarifications. This ability is essential to QA systems, especially for handling ambiguity and facilitating better question understanding. Most of the work in this domain has been driven by extracting tasks from open QA forums [14, 66, 90].

\section{FORMAT AND SUPPORT}

A detailed schedule for our proposed half-day tutorial (three hours plus breaks), which is aimed to meet a high-quality presentation within the chosen time period, is as follows:

- 9:00 - 10:30 Part I: QA over knowledge graphs (1.5 hours)

- Background (15 minutes)

- Simple QA (10 minutes)

- Complex QA (20 minutes)

- Heterogeneous QA (15 minutes)

- Conversational QA (15 minutes)

- Summary (15 minutes)

- 10:30 - 11:00 Coffee break
- 11:00 - 12:30 Part II: QA over textual sources (1.5 hours)

- Background (15 minutes)

- Machine reading (20 minutes)

- Open-domain QA (20 minutes)

- Feedback and interpretability (10 minutes)

- Conversational QA (10 minutes)

- Summary (15 minutes)

\section{PRESENTERS}

Rishiraj Saha Roy is a Senior Researcher at the Max Planck Institute for Informatics (MPII), Saarbrücken, Germany. He leads the research group on Question Answering, that focuses on robust and interpretable solutions for answering natural language questions over structured and unstructured data. He has more than four years of research experience on question answering. In recent years, he has served on the PCs of conferences like SIGIR, CIKM, AAAI, EMNLP, and NAACL, and published at venues like SIGIR, CIKM, WSDM, WWW, and NAACL.

Prior to joining MPII, he worked for one and a half years as a Computer Scientist at Adobe Research, Bangalore, India. He completed his $\mathrm{PhD}$ as a Microsoft Research India Fellow from the Indian Institute of Technology (IIT) Kharagpur. More information about his research can be found at http://people.mpi-inf.mpg.de/ rsaharo/. Avishek Anand is an Assistant Professor at the Leibniz Universität Hannover, Germany. His research interests lie at the intersection of retrieval, mining, and machine learning. He did his $\mathrm{PhD}$ at the Max Planck Institute for Informatics, Saarbrücken, Germany, where he worked on temporal information retrieval. Currently, he is developing intelligent and transparent representation learning methods for text and graphs in the context of search, QA and Web tasks. He has published his research in several top-tier conferences, such as SIGIR, WSDM, CIKM, ICDE and TKDE.

He delivered a tutorial at SIGIR 2016, on Temporal Information Retrieval, and several talks in various summer schools and invited lectures. Avishek was one of the lead organizers of the recently concluded Dagstuhl seminar on Conversational Search.

\section{REFERENCES}

[1] Abdalghani Abujabal, Rishiraj Saha Roy, Mohamed Yahya, and Gerhard Weikum. 2017. QUINT: Interpretable Question Answering over Knowledge Bases. In EMNLP.

[2] Abdalghani Abujabal, Rishiraj Saha Roy, Mohamed Yahya, and Gerhard Weikum. 2018. Never-ending learning for open-domain question answering over knowledge bases. In $W W W$.

[3] Abdalghani Abujabal, Rishiraj Saha Roy, Mohamed Yahya, and Gerhard Weikum. 2019. ComQA: A Community-sourced Dataset for Complex Factoid Question Answering with Paraphrase Clusters. In NAACL-HLT '19.

[4] Abdalghani Abujabal, Mohamed Yahya, Mirek Riedewald, and Gerhard Weikum. 2017. Automated template generation for question answering over knowledge graphs. In $W W W$

[5] Eugene Agichtein, David Carmel, Dan Pelleg, Yuval Pinter, and Donna Harman. 2015. Overview of the TREC 2015 LiveQA Track. In TREC.

[6] Aditya Kalyanpur Alfio Gliozzo and James Fan. 2012. Natural language processing in Watson. In NAACL-HLT.

[7] Avishek Anand, Lawrence Cavedon, Hideo Joho, Mark Sanderson, and Benno Stein. 2020. Conversational Search (Dagstuhl Seminar 19461). Dagstuhl Reports 9, $11(2020)$.

[8] Sören Auer, Christian Bizer, Georgi Kobilarov, Jens Lehmann, Richard Cyganiak, and Zachary Ives. 2007. DBpedia: A nucleus for a Web of open data. (2007).

[9] Junwei Bao, Nan Duan, Zhao Yan, Ming Zhou, and Tiejun Zhao. 2016. Constraint-based question answering with knowledge graph. In COLING. 
[10] Hannah Bast and Elmar Haussmann. 2015. More accurate question answering on Freebase. In CIKM.

[11] Jonathan Berant, Andrew Chou, Roy Frostig, and Percy Liang. 2013. Semantic parsing on Freebase from question-answer pairs. In EMNLP.

[12] Nikita Bhutani, Xinyi Zheng, and HV Jagadish. 2019. Learning to Answer Complex Questions over Knowledge Bases with Query Composition. In CIKM.

[13] Kurt Bollacker, Colin Evans, Praveen Paritosh, Tim Sturge, and Jamie Taylor. 2008. Freebase: A collaboratively created graph database for structuring human knowledge. In SIGMOD.

[14] Pavel Braslavski, Denis Savenkov, Eugene Agichtein, and Alina Dubatovka 2017. What do you mean exactly? Analyzing clarification questions in CQA In CHIIR.

[15] Soumen Chakrabarti. 2020. Interpretable Complex Question Answering. In $W W W$.

[16] Danqi Chen, Adam Fisch, Jason Weston, and Antoine Bordes. 2017. Reading Wikipedia to Answer Open-Domain Questions. In ACL.

[17] Yu Chen, Lingfei Wu, and Mohammed J Zaki. 2019. Bidirectional Attentive Memory Networks for Question Answering over Knowledge Bases. In NAACLHLT.

[18] Eunsol Choi, He He, Mohit Iyyer, Mark Yatskar, Wen-tau Yih, Yejin Choi, Percy Liang, and Luke Zettlemoyer. 2018. QuAC: Question answering in context. In EMNLP.

[19] Philipp Christmann, Rishiraj Saha Roy, Abdalghani Abujabal, Jyotsna Singh, and Gerhard Weikum. 2019. Look before you Hop: Conversational Question Answering over Knowledge Graphs Using Judicious Context Expansion. In CIKM.

[20] Christopher Clark and Matt Gardner. 2018. Simple and Effective MultiParagraph Reading Comprehension. In ACL.

[21] Charles L. A. Clarke and Egidio L. Terra. 2003. Passage retrieval vs. document retrieval for factoid question answering. In SIGIR.

[22] Daniel Cohen, Liu Yang, and W Bruce Croft. 2018. WikiPassageQA: A bench mark collection for research on non-factoid answer passage retrieval. In SIGIR.

[23] Silviu Cucerzan and Eugene Agichtein. 2005. Factoid Question Answering over Unstructured and Structured Web Content.. In TREC.

[24] Jeffrey Dalton, Chenyan Xiong, and Jamie Callan. 2019. CAsT 2019: The con versational assistance track overview. In TREC.

[25] Rajarshi Das, Shehzaad Dhuliawala, Manzil Zaheer, Luke Vilnis, Ishan Durugkar, Akshay Krishnamurthy, Alex Smola, and Andrew McCallum. 2018. Go for a walk and arrive at the answer: Reasoning over paths in knowledge bases using reinforcement learning. In ICLR.

[26] Rajarshi Das, Manzil Zaheer, Siva Reddy, and Andrew McCallum. 2017. Question Answering on Knowledge Bases and Text using Universal Schema and Memory Networks. In $A C L$.

[27] Pradeep Dasigi, Nelson F Liu, Ana Marasovic, Noah A Smith, and Matt Gard ner. 2019. Quoref: A reading comprehension dataset with questions requiring coreferential reasoning. In EMNLP-IFCNLP.

[28] Mostafa Dehghani, Hosein Azarbonyad, Jaap Kamps, and Maarten de Rijke. 2019. Learning to transform, combine, and reason in open-domain question answering. In WSDM.

[29] Laura Dietz, Manisha Verma, Filip Radlinski, and Nick Craswell. 2017. TREC Complex Answer Retrieval Overview. In TREC.

[30] Jiwei Ding, Wei Hu, Qixin Xu, and Yuzhong Qu. 2019. Leveraging Frequent Query Substructures to Generate Formal Queries for Complex Question Answering. In EMNLP-IFCNLP.

[31] Xin Dong, Evgeniy Gabrilovich, Geremy Heitz, Wilko Horn, Ni Lao, Kevin Murphy, Thomas Strohmann, Shaohua Sun, and Wei Zhang. 2014. Knowledge vault: A web-scale approach to probabilistic knowledge fusion. In KDD.

[32] Dheeru Dua, Yizhong Wang, Pradeep Dasigi, Gabriel Stanovsky, Sameer Singh, and Matt Gardner. 2019. DROP: A reading comprehension benchmark requiring discrete reasoning over paragraphs. In NAACL-HLT.

[33] Ahmed Elgohary, Chen Zhao, and Jordan Boyd-Graber. 2018. A dataset and baselines for sequential open-domain question answering. In EMNLP.

[34] Anthony Fader, Luke Zettlemoyer, and Oren Etzioni. 2013. Paraphrase-driven learning for open question answering. In ACL.

[35] Anthony Fader, Luke Zettlemoyer, and Oren Etzioni. 2014. Open question answering over curated and extracted knowledge bases. In $K D D$.

[36] James Fan and Ken Barker. 2015. Natural language processing in Watson. In AAAI.

[37] David Ferrucci, Eric Brown, Jennifer Chu-Carroll, James Fan, David Gondek, Aditya A. Kalyanpur, Adam Lally, J. William Murdock, Eric Nyberg, John Prager, Nico Schlaefer, and Chris Welty. 2010. Building Watson: An overview of the DeepQA project. AI magazine 31, 3 (2010).

[38] Bert F Green Jr, Alice K Wolf, Carol Chomsky, and Kenneth Laughery. 1961 Baseball: An automatic question-answerer. In Western joint IRE-AIEE-ACM computer conference.

[39] Daya Guo, Duyu Tang, Nan Duan, Ming Zhou, and Jian Yin. 2018. Dialog-toaction: Conversational question answering over a large-scale knowledge base. In NeurIPS.
[40] Yangyang Guo, Zhiyong Cheng, Liqiang Nie, Yibing Liu, Yinglong Wang, and Mohan Kankanhalli. 2019. Quantifying and Alleviating the Language Prior Problem in Visual Question Answering. In SIGIR.

[41] Sanda Harabagiu and Dan Moldovan. 2001. Open-domain textual question answering. In NAACL-HLT.

[42] Sen Hu, Lei Zou, Jeffrey Xu Yu, Haixun Wang, and Dongyan Zhao. 2017. Answering natural language questions by subgraph matching over knowledge graphs. TKDE 30, 5 (2017)

[43] Sen Hu, Lei Zou, and Xinbo Zhang. 2018. A state-transition framework to answer complex questions over knowledge base. In EMNLP.

[44] Xiao Huang, Jingyuan Zhang, Dingcheng Li, and Ping Li. 2019. Knowledge graph embedding based question answering. In WSDM.

[45] Mohit Iyyer, Wen-tau Yih, and Ming-Wei Chang. 2017. Search-based neural structured learning for sequential question answering. In $A C L$.

[46] Zhen Jia, Abdalghani Abujabal, Rishiraj Saha Roy, Jannik Strötgen, and Gerhard Weikum. 2018. TEQUILA: Temporal Question Answering over Knowledge Bases. In CIKM.

[47] Mandar Joshi, Uma Sawant, and Soumen Chakrabarti. 2014. Knowledge graph and corpus driven segmentation and answer inference for telegraphic entityseeking queries. In EMNLP.

[48] Magdalena Kaiser, Rishiraj Saha Roy, and Gerhard Weikum. 2020. Conversational Question Answering over Passages by Leveraging Word Proximity Networks. In SIGIR.

[49] Tushar Khot, Ashish Sabharwal, and Peter Clark. 2017. Answering Complex Questions Using Open Information Extraction. In $A C L$.

[50] Bernhard Kratzwald and Stefan Feuerriegel. 2019. Learning from on-line user feedback in neural question answering on the web. In $W W W$.

[51] Tom Kwiatkowski, Jennimaria Palomaki, Olivia Redfield, Michael Collins, Ankur Parikh, Chris Alberti, Danielle Epstein, Illia Polosukhin, Jacob Devlin, and Kenton Lee. 2019. Natural questions: A benchmark for question answering research. TACL 7 (2019).

[52] Patrick Lewis, Ludovic Denoyer, and Sebastian Riedel. 2019. Unsupervised Question Answering by Cloze Translation. In ACL.

[53] Fei Li and HV Jagadish. 2014. Constructing an interactive natural language interface for relational databases. In $V L D B$.

[54] Jimmy Lin and Boris Katz. 2003. Question answering techniques for the World Wide Web. In EACL.

[55] Yankai Lin, Haozhe Ji, Zhiyuan Liu, and Maosong Sun. 2018. Denoising distantly supervised open-domain question answering. In $A C L$.

[56] Xiaolu Lu, Soumajit Pramanik, Rishiraj Saha Roy, Abdalghani Abujabal, Yafang Wang, and Gerhard Weikum. 2019. Answering Complex Questions by Joining Multi-Document Evidence with Quasi Knowledge Graphs. In SIGIR.

[57] Kangqi Luo, Fengli Lin, Xusheng Luo, and Kenny Zhu. 2018. Knowledge Base Question Answering via Encoding of Complex Query Graphs. In EMNLP.

[58] Hao Ma and Yan Ke. 2015. An introduction to entity recommendation and understanding. In $W W W$.

[59] Mausam. 2016. Open information extraction systems and downstream applications. In IfCAI.

[60] Anusri Pampari, Preethi Raghavan, Jennifer Liang, and Jian Peng. 2018. emrQA: A Large Corpus for Question Answering on Electronic Medical Records. In EMNLP.

[61] Boyuan Pan, Hao Li, Ziyu Yao, Deng Cai, and Huan Sun. 2019. Reinforced Dynamic Reasoning for Conversational Question Generation. In ACL.

[62] Panupong Pasupat and Percy Liang. 2015. Compositional Semantic Parsing on Semi-Structured Tables. In ACL.

[63] Yunqi Qiu, Yuanzhuo Wang, Xiaolong Jin, and Kun Zhang. 2020. Stepwise Reasoning for Multi-Relation Question Answering over Knowledge Graph with Weak Supervision. In WSDM.

[64] Filip Radlinski and Nick Craswell. 2017. A theoretical framework for conversational search. In CHIIR.

[65] Pranav Rajpurkar, Jian Zhang, Konstantin Lopyrev, and Percy Liang. 2016. SQuAD: 100,000+ Questions for Machine Comprehension of Text. In EMNLP.

[66] Sudha Rao and Hal Daumé III. 2018. Learning to ask good questions: Ranking clarification questions using neural expected value of perfect information. In $A C L$.

[67] Deepak Ravichandran and Eduard Hovy. 2002. Learning surface text patterns for a question answering system. In $A C L$.

[68] Siva Reddy, Danqi Chen, and Christopher Manning. 2019. CoQA: A conversational question answering challenge. TACL 7 (2019).

[69] Marzieh Saeidi, Max Bartolo, Patrick Lewis, Sameer Singh, Tim Rocktäschel, Mike Sheldon, Guillaume Bouchard, and Sebastian Riedel. 2018. Interpretation of Natural Language Rules in Conversational Machine Reading. In EMNLP.

[70] Amrita Saha, Vardaan Pahuja, Mitesh Khapra, Karthik Sankaranarayanan, and Sarath Chandar. 2018. Complex sequential question answering: Towards learning to converse over linked question answer pairs with a knowledge graph. In AAAI.

[71] Denis Savenkov and Eugene Agichtein. 2016. When a knowledge base is not enough: Question answering over knowledge bases with external text data. In 
SIGIR

[72] Uma Sawant, Saurabh Garg, Soumen Chakrabarti, and Ganesh Ramakrishnan 2019. Neural architecture for question answering using a knowledge graph and Web corpus. Information Retrieval fournal (2019).

[73] Tao Shen, Xiubo Geng, QIN Tao, Daya Guo, Duyu Tang, Nan Duan, Guodong Long, and Daxin Jiang. 2019. Multi-Task Learning for Conversational Question Answering over a Large-Scale Knowledge Base. In EMNLP-I7CNLP.

[74] Fabian Suchanek, Gjergji Kasneci, and Gerhard Weikum. 2007. YAGO: A core of semantic knowledge. In $W W W$.

[75] Haitian Sun, Tania Bedrax-Weiss, and William Cohen. 2019. PullNet: Open Domain Question Answering with Iterative Retrieval on Knowledge Bases and Text. In EMNLP-IFCNLP.

[76] Haitian Sun, Bhuwan Dhingra, Manzil Zaheer, Kathryn Mazaitis, Ruslan Salakhutdinov, and William Cohen. 2018. Open Domain Question Answering Using Early Fusion of Knowledge Bases and Text. In EMNLP.

[77] Huan Sun, Hao Ma, Xiaodong He, Wen-tau Yih, Yu Su, and Xifeng Yan. 2016 Table cell search for question answering. In $W W W$.

[78] H. Sun, H. Ma, W. Yih, C. Tsai, J. Liu, and M. Chang. 2015. Open domain question answering via semantic enrichment. In $W W W$.

[79] Alona Sydorova, Nina Poerner, and Benjamin Roth. 2019. Interpretable Question Answering on Knowledge Bases and Text. In $A C L$.

[80] A. Talmor and J. Berant. 2018. The Web as a Knowledge-base for Answering Complex Questions. In NAACL-HLT.

[81] Alon Talmor and Jonathan Berant. 2019. MultiQA: An empirical investigation of generalization and transfer in reading comprehension. In $A C L$

[82] C. Tan, F. Wei, Q. Zhou, N. Yang, B. Du, W. Lv, and M. Zhou. 2018. ContextAware Answer Sentence Selection With Hierarchical Gated Recurrent Neural Networks. IEEE/ACM Trans. Audio, Speech \& Language Processing 26, 3 (2018).

[83] Christina Unger, Lorenz Bühmann, Jens Lehmann, Axel-Cyrille Ngonga Ngomo, Daniel Gerber, and Philipp Cimiano. 2012. Template-based question answering over RDF data. In $W W W$.

[84] Svitlana Vakulenko, Javier David Fernandez Garcia, Axel Polleres, Maarten de Rijke, and Michael Cochez. 2019. Message Passing for Complex Question Answering over Knowledge Graphs. In CIKM.

[85] Ellen M. Voorhees. 1999. The TREC-8 question answering track report. In TREC

[86] Denny Vrandečić and Markus Krötzsch. 2014. Wikidata: A free collaborative knowledge base. CACM 57, 10 (2014).
[87] Bingning Wang, Ting Yao, Oi Zhang, Jingfang Xu, Zhixing Tian, Kang Liu, and Jun Zhao. 2019. Document Gated Reader for Open-Domain Question Answering. In SIGIR.

[88] Zhiyong Wu, Ben Kao, Tien-Hsuan Wu, Pengcheng Yin, and Oun Liu. 2020. PERQ: Predicting, Explaining, and Rectifying Failed Questions in KB-QA Systems. In WSDM.

[89] Wenhan Xiong, Mo Yu, Shiyu Chang, Xiaoxiao Guo, and William Yang Wang. 2019. Improving Question Answering over Incomplete KBs with KnowledgeAware Reader. In $A C L$.

[90] Jingjing Xu, Yuechen Wang, Duyu Tang, Nan Duan, Pengcheng Yang, Qi Zeng, Ming Zhou, and Xu Sun. 2019. Asking Clarification Questions in KnowledgeBased Question Answering. In EMNLP-IFCNLP.

[91] Kun Xu, Yuxuan Lai, Yansong Feng, and Zhiguo Wang. 2019. Enhancing KeyValue Memory Neural Networks for Knowledge Based Question Answering. In NAACL-HLT.

[92] Kun Xu, Siva Reddy, Yansong Feng, Songfang Huang, and Dongyan Zhao. 2016. Question Answering on Freebase via Relation Extraction and Textual Evidence. In $A C L$.

[93] Mohamed Yahya, Klaus Berberich, Shady Elbassuoni, Maya Ramanath, Volker Tresp, and Gerhard Weikum. 2012. Natural language questions for the web of data. In EMNLP.

[94] Mohamed Yahya, Klaus Berberich, Shady Elbassuoni, and Gerhard Weikum. 2013. Robust question answering over the web of linked data. In CIKM.

[95] Yunlun Yang, Yu Gong, and Xi Chen. 2018. Query Tracking for E-commerce Conversational Search: A Machine Comprehension Perspective. In CIKM

[96] Zhilin Yang, Peng Qi, Saizheng Zhang, Yoshua Bengio, William Cohen, Ruslan Salakhutdinov, and Christopher D Manning. 2018. HotpotQA: A Dataset for Diverse, Explainable Multi-hop Question Answering. In EMNLP.

[97] Scott Wen-tau Yih and Hao Ma. 2016. Question answering with knowledge base, Web and beyond. In NAACL-HLT.

[98] Wen-tau Yih and Hao Ma. 2016. Question answering with knowledge base, Web and beyond. In SIGIR.

[99] Pengcheng Yin, Nan Duan, Ben Kao, Junwei Bao, and Ming Zhou. 2015. Answering questions with complex semantic constraints on open knowledge bases. In CIKM.

[100] Xinbo Zhang, Lei Zou, and Sen Hu. 2019. An Interactive Mechanism to Improve Question Answering Systems via Feedback. In CIKM.

[101] Kangyan Zhou, Shrimai Prabhumoye, and Alan W Black. 2018. A Dataset for Document Grounded Conversations. In EMNLP. 